\title{
A Web-Based Flexible Communication System in Radiology
}

\author{
Alexander Valentine Rybkin • Mark Wilson
}

Published online: 9 December 2010

(C) The Author(s) 2010. This article is published with open access at Springerlink.com

\begin{abstract}
A web-based system for rapid multidirectional communication has been created in the Radiology department at San Francisco General Hospital. The system allows messaging among radiology attendings, residents, and technologists, as well as other members of the hospital community, such as Emergency Department physicians and nurses. Instead of being tied to a particular workflow, this system provides a flexible communication infrastructure which can be easily adapted for different functions and user roles. The system has so far been configured to successfully support the standard "wet reading" workflow, to support marking and tracking of critical results, as well as multiple educational and quality improvement workflows. In the 19 months of operation, the system has gained over 1,800 users (virtually all providers at our institution), it has been accessed by radiologists over 39,000 times and by nonradiologists over 34,000 times. It has become an integral part of the radiology department operations and nonradiology clinical workflows. Unlike most existing softwares, our system is not a task-specific application, but a multipurpose communication system. It is able to effectively accommodate multiple workflows and user roles through configuration (without additional programming). This flexibility has helped this system to be rapidly and widely adopted within our enterprise. The extended reach of the system enables improved monitoring and documentation of workflows, helping with management decision making, and quality assurance. We report a successful radiology communication
\end{abstract}

\section{A. V. Rybkin $(\bowtie) \cdot$ M. Wilson}

Department of Radiology and Biomedical Imaging,

San Francisco General Hospital, University of California,

San Francisco,

1001 Potrero Ave Rm 1x57,

San Francisco, CA 94110, USA

e-mail: manxray@yahoo.com system based on the principles of flexibility and inclusiveness of users inside and outside the radiology department.

Keywords Administration - Auditing - Clinical information systems · Clinical workflow Communication Computer communication networks - Computerized method - Critical results reporting - Efficiency - Electronic medical record (EMR) - Workflow · Hospital information systems (HIS) . Radiology workflow - Medical records system . Computerized - Quality assurance

\section{Introduction}

In the age when many Radiology departments become computerized, many aspects of a radiologist's workflow rely on word of mouth, pen and paper, telephone, and fax. Radiologists use these ad hoc communication strategies when they deliver important findings, when they interact with support personnel, such as technologists and nurses, or when they help determine the priority of studies to be done. These communications have limited scope (they are usually person-to-person), they are difficult to document and track; they distract radiologists from the task of image interpretation, causing workflow delays and reduced productivity.

Several reports have outlined attempts to replace informal communication with internet-based systems [1-4]. Most of them concentrate on communication of "wet readings" between on-call radiologists and referring healthcare providers. These systems exemplify the conventional view of radiological communication as a "loop," in which information flows in the orderly fashion from the provider to the radiologist (in the form of a request) and back to the originator (in the form of a "wet reading") [5]. However, the reality of the radiology department in a busy hospital does 
not fit this simplified model. Information about any particular study flows to the on-call radiologist from multiple directions. Multiple members of the emergency or trauma surgery team may contribute crucial pieces of historical information. Nurses and technologists relate the specifics of technique and of adverse events. The radiologist, in addition to providing "wet readings", directs the workflow by assigning priorities to requested studies, determines protocols, and provides feedback to the staff on the studies' quality. Most of these interactions occur informally because they are not anticipated by a system where the roles and the direction information flow are rigidly defined. In contrast, one can reach virtually anyone by phone, fax, or pager. The content of a conversation (either by telephone or face to face) is not constrained by the underlying technology. The key advantage of the informal means of communication is their flexibility.

In developing our own internet-based communication system, we made flexibility the primary goal of design. We consequently found the system useful for multiple elements of radiology workflow that had previously been handled informally. The inherent flexibility or our system also allows us to extend it in ways we did not anticipate at the beginning.

Our system, codenamed Radiologue, consists of a web application running on the hospital intranet. For the convenience of radiologists, our system can be accessed from the departmental picture archiving and communication system (PACS). Healthcare providers and support personnel access Radiologue through the hospital-wide information system and electronic medical record (Siemens LCR/Invision). Managers can access the web application directly from the web browser. Radiologue supports multiple modes of role-aware user authentication to ensure that only authorized users have access to protected health information. The result of this arrangement is that access to the system is secure and ubiquitous throughout the healthcare enterprise.

Radiologue application provides a web portal, which includes a configurable worklist of current radiological studies. The users of the system are invited to enter messages about these radiological exams. The messages (or "notes") are highly configurable, and can take the form of a "wet read", study protocol, referring physician's request for specific information, or a comment by the technologist who performs the study. The ability to configure and extend the messages provides the flexibility to use them in different workflows. Our application has become a tool for collaborative management of radiological cases, which allows multidirectional data exchange. We believe that such architecture improves upon the standard model of the unidirectional "communication loop" of healthcare information exchange. A collaborative multidi- rectional information exchange model is more akin to a "blog" or a "wiki" than to a traditional repository of medical information.

\section{Methods}

The web application resides on a HP Proliant 5200 server (Hewlett Packard, Palo Alto, CA) running Windows Server 2003 R2 operating system (Microsoft, Redmond, WA), which includes the Internet Information Services web server software. The application was developed using C\# programming language using ASP.NET 2.0 framework. This technology has a proven track record in development of enterprise-level web software. It has rich support for interactions with databases and XML; it has advanced tools for creation of object-relational mapping, and it emphasizes modern web design techniques, including Web Services and Asynchronous Javascript and XML (AJAX). Perhaps more importantly, Microsoft technology is ubiquitous throughout medical information systems, and has the advantage of familiarity and supportability by most IT personnel. Using proven technology helped us convince the hospital administration to adopt our experimental system.

The database layer of our application consists of SQL Server 2005 (Microsoft, Redmond, WA), which is the preferred database for ASP.NET technology. This database software provides a rich and well-documented set of features that ensure logical integrity of the data within the application. It is tightly integrated with the development environment (Visual Studio 2005) and provides tools for simplified administration tasks, such as data backup.

Figure 1 provides a schematic representation of the application's architecture. The web server resides on the hospital intranet. It periodically queries the radiology information system (RIS) via a standard ODBC-compliant database connection to retrieve information about the current radiological studies. The web application displays this information to the end user in the form of a customizable worklist, which is displayed in the web browser (Fig. 2). The studies on the worklist are initially ordered chronologically, but they can be rearranged by the end user to reflect clinical priority. The updated worklist is constantly published to the other users of the system. Such continuous communication between the central application server and client browser is made possible by AJAX technology, which is used in the modern blogging and instant messaging systems.

In addition to reordering the worklist, the users can add information to the studies on the worklist in the form of notes (Fig. 3). A "note" is the central feature of the Radiologue application, as it is responsible for the system's flexibility. A note is similar to a "topic" in the context of a 


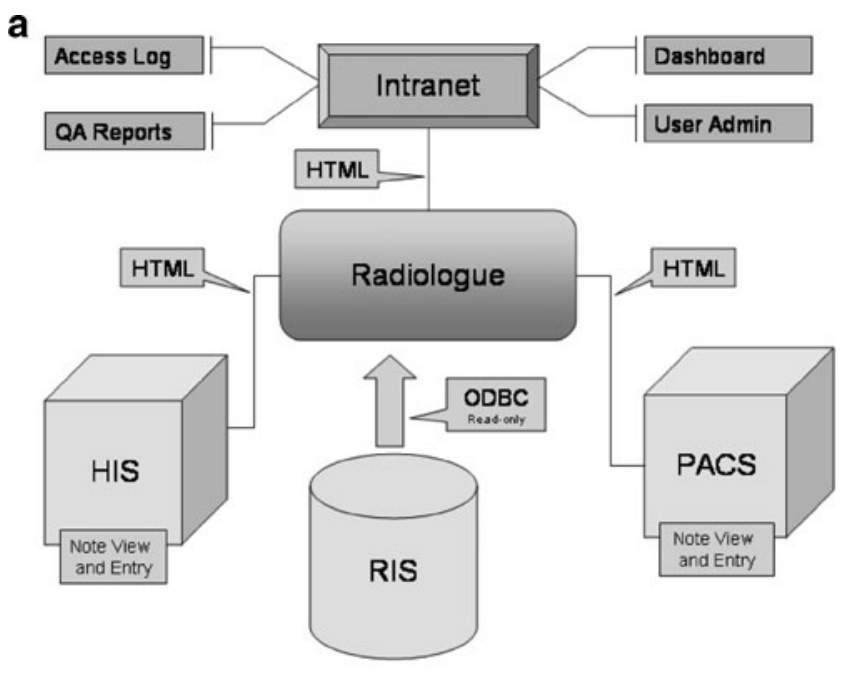

b

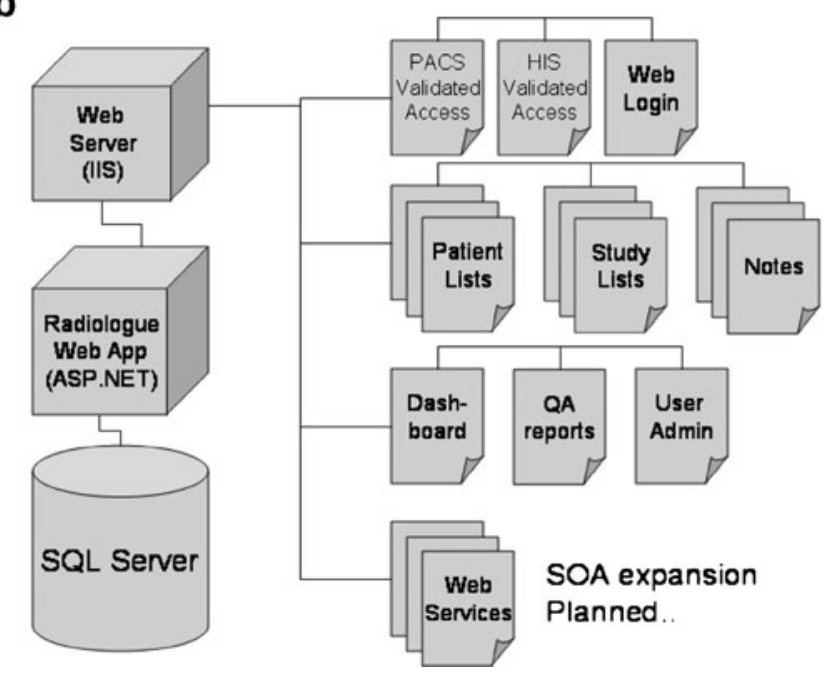

Fig. 1 Schematic representation of the Radiologue web application's architecture. a External connections. The application is connected to the RIS database through a generic ODBC connection. It is integrated into HIS/EMR and into PACS through HTML links using vendor-specific web interfaces. It can also be accessed directly on the intranet through the browser. b Internal architecture. Radiologue is an ASP.NET application that utilizes SQL Server as a database engine. Radiologue serves dynamically generated $\mathrm{html}$ pages, which are specific to the source of connection (HIS/EMR vs. PACS vs. browser) and to the users' role (radiology resident vs. ER attending vs. technologist)

blog or a "conversation" in an instant messaging system. Each note of a certain type is permanently associated with a radiological study, and is displayed in the worklist as a color-coded button. Users contribute to notes collaboratively (however, the application tightly controls who can edit or view each note). Although all notes share basic features and are treated by the application in similar ways, they can also be customized to reflect differences in function. For example, when a note is configured as a "wet reading", it accepts changes only from the on-call radiologists, and is displayed to referring healthcare providers while it is hidden from others. The note's display properties can be set up to reflect the urgency of information it contains. A "wet reading" containing a critical result can be displayed as a bright-red button on the Emergency physician's worklist. Further, a critical wet reading may be configured to page the ordering physician if it is not viewed in a certain time interval.

By using the Radiologue system, one can define arbitrarily many note types and user roles. Such system is highly adaptable to differences of workflow among institutions. It can also grow to encompass additional aspects or radiology workflow as more user and note types are added after initial implementation.

As notes are modified and viewed by the users of the system, all access time-stamped and logged by the application (Fig. 4). Such logging fulfills Health Insurance Portability and Accountability Act (HIPAA) requirements, but, in addition, the resulting database is constantly mined for important operational data which is fed back to the users. This data are graphically represented in the form of a dashboard, which is available to the users who are configured as managers.

\section{Results}

Table 1 demonstrates the roles and notes we defined at our institution. For the initial implementation of the Radiologue system, we have chosen to create a "wet reading" system. A purpose-built wet reading system already existed elsewhere in our institution, and the radiologists (residents and attendings) were already familiar with the wet reading workflow. On-call residents and fellows use this system to communicate their impressions to the healthcare providers regarding CTs and MRIs performed at night on weekdays and on weekends. The residents enter their "wet readings" on a web form accessible directly from their PACS stations. The form allows the residents to mark the urgency of the note as Critical, Medium Priority, and Low Priority (which is the default setting). Healthcare providers (residents and attendings in the Emergency Department) access the system directly from the Electronic Medical Record (EMR), which displays the worklist of pending studies for their patient. Healthcare providers access "wet readings" by clicking on color-coded buttons next to the study of interest. Critical results are displayed in bright-red color, to attract attention.

Attending radiologists participate in the "wet reading" system by entering "review" notes. When entering a "review", attending radiologists have four options to rate the level of disagreement with the "wet reading" note: "Agree", "Minor Discrepancy", "Major Discrepancy", and "Good Job". When there is a disagreement, attending radiologists provide correct interpretations and teaching 
Fig. 2 Worklist view of the active studies. Studies to be annotated are displayed in a customizable worklist. Various notes are displayed to the right of the study demographic information as color-coded buttons. The notes can be immediately accessed by clicking on the buttons

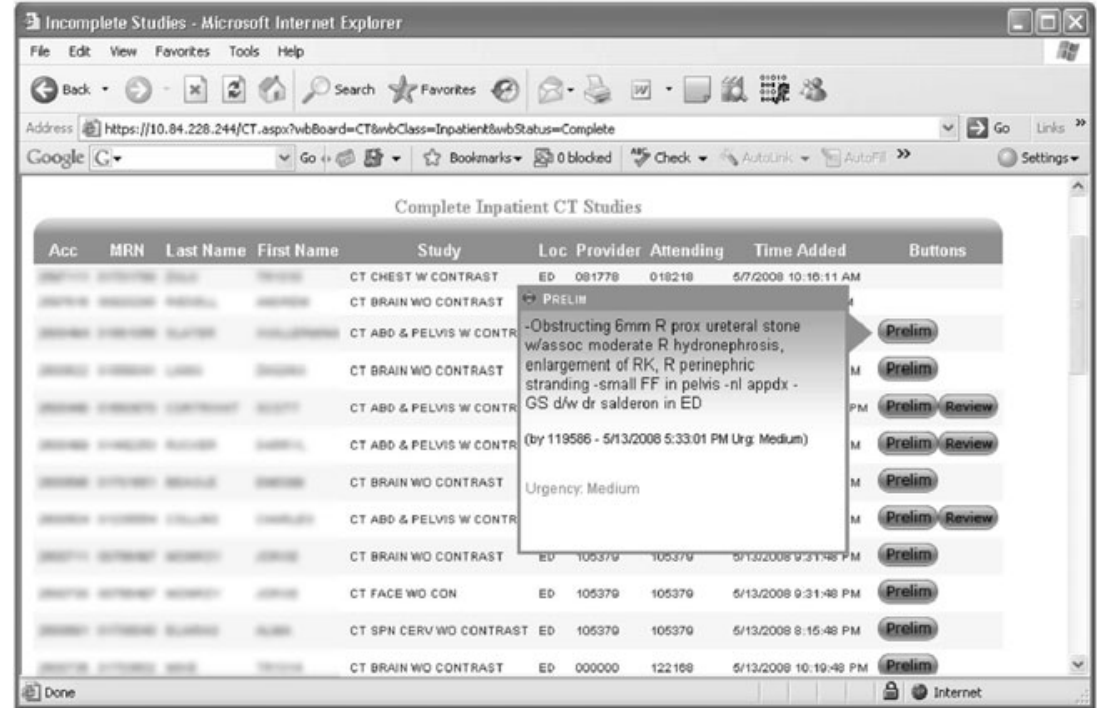

points in the text of the review note. The application displays these notes to the healthcare providers in a similar fashion to the "wet reading" notes. Reviews with "Major Discrepancy" appear on the worklist as bright-red buttons.

The application contains a Quality Assurance function, which allows residents to query the wet readings with which the attending radiologists disagreed, and to read the associated "review" notes. This educational function helps residents learn from their mistakes. This function is utilized both for individual learning and in the formal Resident Quality Assurance conference, which is held at our institution every month. The log of time-stamped information access, which our system generated, has also been very helpful in discussions of problem cases in the setting of hospital-wide Trauma Quality Assurance conferences. Usually, a clear sequence of events (including timing, content, and recipient of communication) can be reconstructed from these logs. We found such clarity helpful in defending radiologists' actions.

The data collected in the Radiologue application constitute records of the formal quality assurance proceedings, therefore, its discovery in the court of law is prohibited
Fig. 3 Note input window configured for "Tech note" communication. Notes can be also configured for

"Wet reading", "History",

"Protocol", "Nurse note", "ED note", and many others. Appropriate notes are displayed for each user role. For example, Tech notes are displayed only to radiologists and technologists, and History notes are displayed to everybody, including referring physicians and nurses. The urgency of each note can be marked as "Low", "Medium", or "Critical"
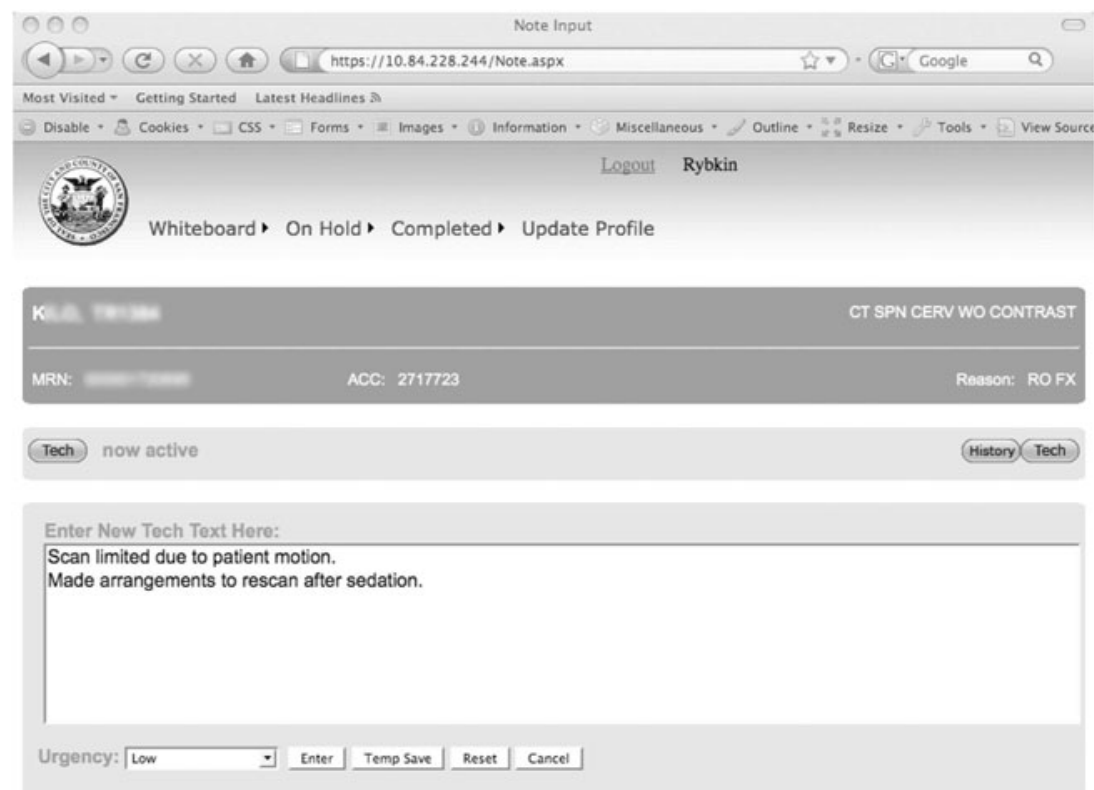
Fig. 4 Access log page. All actions performed by the users or by the system are logged in the database. Logging ensures HIPAA compliance, allows QA reviews, and dashboard functions. Logging information is accessed through a specialized interface that allows searching and sorting on multiple demographic parameters

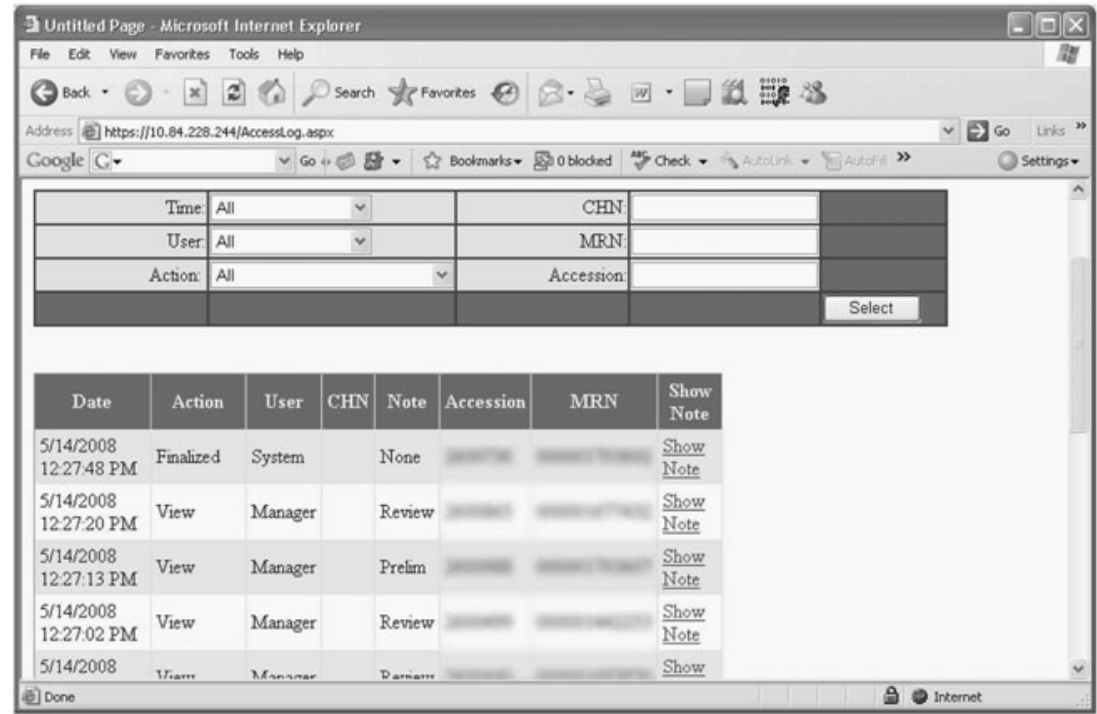

under California Evidence Code Section 1157. In addition to security measures mandated by legislation governing protected health information (HIPAA), the application includes several additional measures to prevent accidental discovery of sensitive quality assurance information. Although the application utilizes identifying information to keep track of the cases, for the purposes of presentation, the identifying data are stripped. Also, outside the quality assurance context, the "wet reading" and "review" notes are hidden from users when the final report for the radiological exam is entered into the medical record. This final report, which is usually dictated by a resident or fellow and approved by an attending radiologist, constitutes the ultimate diagnostic result and the summary of preceding communication.

The application incorporates a dashboard, which displays workflow information derived from the access log (Fig. 5). Access to this dashboard is strictly limited to the departmental management. Numbers of critical results, major disagreements with wet readings, and time delay in providing wet readings are tracked and graphed in real time. Because the application utilizes tracking data from the RIS, the dashboard can calculate and display operational derivatives, such as throughput information. Patientspecific protected health information is stripped from all data used for management purposes.

As of this writing, Radiologue has been in continuous operation for 19 months. It has been readily adopted by the Radiology staff and by the clinicians. Currently, the system has 1,822 users, including 149 radiologists (residents rotating 14 at any one time, fellows rotating seven at any one time, and 14 attendings), 13 radiology managers (which include chief technologists), and 1,650 clinicians (residents, attendings, and nurses). The system has been accessed 39,674 times by radiologists and 34,926 times by clinicians.

\section{Discussion}

Computerized systems for communication exchange are becoming widespread. By integrating a formal reporting
Table 1 The roles defined within Radiologue, with their associated note types and permitted actions

A user in a particular role can create and nondestructively edit the corresponding notes. Rolespecific permissions can be set for viewing the notes. Actions represent functions, which are not tied to specific notes, such as management and QA functions

\begin{tabular}{lll}
\hline Role & Notes & Actions \\
\hline Attending radiologist & $\begin{array}{c}\text { Preliminary read, review read, } \\
\text { history note, protocol note, } \\
\text { interesting case }\end{array}$ & Change study priority, QA view \\
Resident radiologist & $\begin{array}{l}\text { Priminary read, history note, } \\
\text { protocol note }\end{array}$ & QA view \\
ER attending & $\begin{array}{l}\text { History note, ER note } \\
\text { Clinical MD }\end{array}$ & Change study priority \\
Nurse & History note, MD note & None \\
Technologist & History note, Tech note & $\begin{array}{l}\text { None } \\
\text { Change study priority }\end{array}$ \\
Manager & None & $\begin{array}{l}\text { User administration, access log, departmental } \\
\text { dashboard, critical result report, QA report, } \\
\text { new roles, new notes }\end{array}$ \\
\hline
\end{tabular}


Fig. 5 Dashboard page. This feature allows real-time monitoring of key performance indicators gleaned or calculated from the action logging database. Dashboard is currently used to monitor the number of critical results, number of major discrepancies between resident wet reads and final results, and timing of the wet reads among other performance indicators

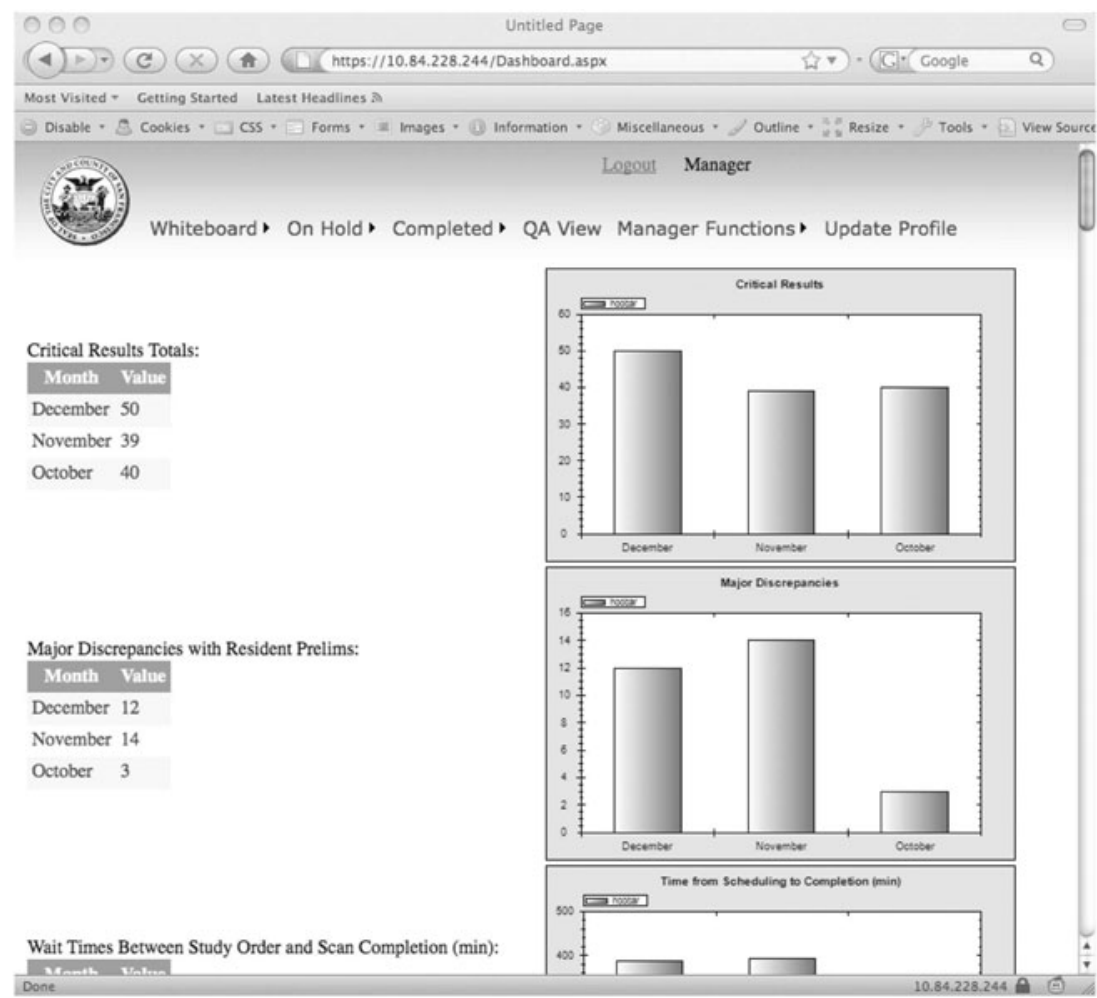

system in the workflow of on-call residents, several academic institutions were able to enhance timely reporting and to monitor interpretation errors [1]. An electronic message-based system has been described for clinical communication with an off-site radiologist [4]. Others have combined reporting capability with elements of physician order entry and workflow management [6]. Nagy at al. report an electronic communication among radiologists and support personnel for tracking technical problems [4] and for improving quality assurance procedures [7]. Bland et al. describe a web-based application, which facilitates exchange of information among clinicians and scientists in research environment [8]. The multitude of applications dedicated to electronic communication reflects the complexity of radiology workflow. Radiologists need to communicate with various members of the healthcare team, for a variety of purposes, under a variety of conditions. Therefore, we believe that there is a need for a unified electronic communication system that would be flexible enough to accommodate a multitude of uses.

The Radiologue system is our first attempt at creation of such an inclusive, pleuripotent system. Radiologue allows participation by multiple kinds of users (doctors, nurses, and technologists) in ways that can be tailored to their roles. The system enables one to quickly and broadly disseminate important information, which is impossible to do through informal means, such as telephone, fax, or any other pointto-point means of communication. Further, Radiologue allows documentation and tracking of information exchange, which result in better quality control and management decisions.

Radiologue has already become an integral part of workflows used by radiologists, technologists, managers, and many clinicians. In implementing Radiologue, we have not changed those workflows. Radiologue was flexible enough to adapt to the existing ways of doing business, while offering new tools to improve efficiency and transparency of the processes. As a result, all stakeholders could easily grasp the advantages of Radiologue from the beginning, facilitating buy-in and simplifying implementation.

The aim of future development of Radiologue is to facilitate and widen radiology-related communication. Modality-specific worklists will be displayed in the various patient care areas of the hospital (nursing stations, emergency department, and scanning areas) on widescreen monitors. These lists are already available to the clinical personnel through EMR integration. We anticipate that such displays will result in better situational awareness by radiology and clinical staff, and ultimately in better modality throughput. We are also involving clinicians in collaborative management of the workflow in our department. In so doing, we are exploring the effects of multidirectional communications between the radiology department and the rest of the healthcare enterprise. Our future efforts will make Radiologue more standardcompliant and portable to other healthcare settings. We 
are also working on an instant messaging module that will facilitate timely critical result distribution.

The legal status of information within Radiologue is a matter of great concern and debate. We believe that we have not sacrificed privacy and security of the protected health information in our striving for openness. In conjunction with our hospital administration, we have decided to treat all information in Radiologue as a part of Medical Record. We therefore maintain commensurate security and backup policies. Similarly to the rest of the medical information, we treat the data used in our Quality Assurance program confidentially.

In designing Radiologue to be flexible and inclusive, we sought to emulate the contemporary web-based technologies, which have collectively become known as "Web 2.0". These applications, which include Google, Wikipedia, Flickr and many others, encourage users to actively participate in creation of content. Through inviting wide participation, these applications created additional value for their users, and thereby challenged the dominance of traditional means of publishing and communication [9]. We firmly believe that this strategy is applicable to Radiology. Our starting point is the realization that healthcare providers, who are outside the traditional communication loop, have important information to contribute to the radiological care of the patient. These users also have important information needs that, if satisfied, can enhance the overall care of the patient. The purpose of any medical communication systems is to break down barriers to effective information sharing. It is therefore imperative that the information systems of the future be designed with sufficient flexibility, in order to avoid creating artificial barriers where none existed before.
Open Access This article is distributed under the terms of the Creative Commons Attribution Noncommercial License which permits any noncommercial use, distribution, and reproduction in any medium, provided the original author(s) and source are credited.

\section{References}

1. Tellis WM, Andriole KP: Integrating Multiple Clinical Information Systems using the Java Message Service Framework. Journal of Digital Imaging 17(2):80-86, 2004

2. Morioka C, Dionisio JD, Bui A, El-Saden S, Kangarloo H: StructConsult: structured real-time wet read consultation infrastructure to support patient care. Stud Health Technol Inform 129(Pt 1): 429-33, 2007

3. Arnold CW, Bui AAT, Morioka C, El-Saden S, Kangarloo H: A Prototype Web-based Reporting System for Onsite-Offsite Clinician Communication. RadioGraphics 27:1201-1211, 2007

4. Nagy P, Warnock M, Daly M, Rehm J, Ehlers K: Radtracker: A Web-Based Open-Source Issue Tracking Tool. Journal of Digital Imaging 15(Suppl 1):114-119, 2002

5. Reiner B: Automating Radiologist Workflow Part 1: The Digital Consultation. J Am Coll Radiol 5:1080-1085, 2008

6. Juluru K, Eng J: Internet-Based Radiology Order-Entry, Reporting, and Workflow Management System for Coordinating Urgent Study Requests During Off-Hours. AJR 184:1017-1020, 2005

7. Nagy PG, Pierce B, Otto M, Safdar NM: Quality Control Management and Communication Between Radiologists and Technologists. JACR 5(6):759-765, 2008

8. Bland PH, Laderach GE, Meyer CR: A Web-based Interface for Communication of Data between the Clinical and Research Environments without Revealing Identifying Information. Acad Radiol 14(6):757-764, 2007

9. What Is Web 2.0 Design Patterns and Business Models for the Next Generation of Software, www.oreilynet.com, 09/30/2005 at http:// www.oreillynet.com/pub/a/oreilly/tim/news/2005/09/30/what-isweb-20.html?page $=1$ 